\title{
La théorie des ondes liquides de gravité dans un bassin tournant
}

\section{Theory of liquid gravity waves in a rotating basin}

\author{
PAR B. SAINT-GUILY,
}

SOYS-DIRECTEUR AU LABORATORE D'OCÉANOGRAPHIE PHYSIQUE DU MUSÉUA D"HISOIRE NATURETIAE

\begin{abstract}
Les etudes thériques consacrées aux ondes liquides de gravité dans un miliea tournant reposent généralement sul l'approximation dite «des ondes longues». M. Brillouin et J. Coulomb se sont libérés de celte approximation dans un travail sur les oscillations d'un liquide pesant dans un bassin cylindrique en rotation. Ils ont ainsi montré l'existence et l'importance des ondes internes ou stratifies en milieu homogène. En se placant dans les mêmes conditions, l'autear examine l'action de l'accélération de Coriolis sur la forme et la propagation des ondes dans un domaine illimité. L'équation d'onde et les conditions aux frontieres sont ecrites. On en donne ensuite des solutions périodiques représentant les ondes libres à orètes horizontales, les ondes produites par un batteur et les ondes libres dans un canal. Ces ondes ont un caractere différent pour des périodes inférieures ou supérieures a la période pendulaire.
\end{abstract}

\begin{abstract}
Theoretical studies on the subject of liquid grabity waves on a rotating medium are usually based on the "long wave" approximation. In their work on the oscillations of a ponderable liquid in a rotating cylindrical tank, M. Brillonin and J. Coulomb were able to disregard this approximation; they thus showed up the existence and importance of internal or stralified waves in a homogeneous medium. Starting out from, the same conditions, the Author eamines the effect of Coriolis aceleration upon the shape and propagation of waves in an infinite area. The wave equation and boundary conditions are then written down and periodic solutions are given for free waves with horigontal crests, waves generated by a paddle, and jree waves in a canal. The characteristics of these waves differ depending on whether their period is less or greater than the basic period.
\end{abstract}

La théorie des ondes de marée, des ondes de tempête et des tsunamis repose sur deux hypothèses principales. La première est celle des petits mouvements; elle permet de linéariser le problème en se limitant au premier ordre. La seconde hypothèse est celle d'une profondeur: petite devant le rayon de courbure de la surface libre ou devant la longueur d'onde; elle conduit, en supposant la pression quasi-hydrostatique et en faisant la moyenne des équations du mouvement et de la vitesse sur toute la profondeur, à une équation d'onde bidimensionnelle relativement simple.

Cette théorie des ondes longues est double- ment restrictive. Elle ne convient évidemment pas à la représentation des courtes longueurs d'onde, et elle présente l'inconvénient de laisser échapper une partie du phénomène étudié : elle ignore en effet l'existence d'ondes internes ou stratifiées qui jouent un ròle important dans un bassin tournant et dans les océans en raison de l'accélération de Coriolis. M. Brillouin et J. Courlomb [1] ont les premiers attiré l'attention sur ce point dans leur mémoire sur les oscillations d'un liquide pesant dans un bassin eylindrique en rotation. Parallèlement, il nous a semblé intéressant d'examiner les solutions périodiques élémentaires représentant des ondes libres, puis lagitation produite par un batteur. 


\section{EQUATION D'ONDE DANS UN BASSIN TOURNANT}

Considérons un fluide parfait, incompressible et de densité constante, et supposons les forces de masses égales au gradient d'une fonction potentielle - $g z-\Gamma$, où $g$ désigne l'accélération de la pesanteur et - $\mathrm{I}$ un potentiel periurbateur quelconque. Les équations du mouvement peuvent alors s'écrire sous la forme:

$$
\begin{aligned}
\frac{\partial \vec{u}}{\partial t}+(\operatorname{rot} u+2 \vec{\omega}) \Delta \vec{u} & =\operatorname{grad} \frac{\partial \Phi}{\partial t} \\
\frac{\partial \Phi}{\partial t}+\frac{u^{2}}{2}+\frac{p}{\rho}+g z+\Gamma & =0, \\
\operatorname{div} u & =0,
\end{aligned}
$$

où la fonction $\Phi$ est définie par la seconde relation (1). Si $\zeta$ désigne la dénivellation de la surface libre par rapport à la surface au repos $(z=0), p_{a}$ la pression atmosphérique et $h$ la profondeur, les conditions sur les frontières surface et fond sont les suivantes:

$$
\begin{aligned}
& \left|\frac{\partial \zeta}{\partial t}+u \frac{\partial \zeta}{\partial x}+v \frac{\partial \zeta}{\partial y}-w\right|_{z=\zeta}=0, \\
& \left|\frac{\partial \Phi}{\partial t}+\frac{u^{2}}{2}+\frac{p_{n}}{\varphi}+g z+\Gamma\right|_{z=\zeta}=0 \\
& \left|u \frac{\partial h}{\partial x}+v \frac{\partial h}{\partial y}+w\right|_{z=-\eta}=0
\end{aligned} \mid
$$

La première condition exprime la coïncidence des vitesses normales de la surface libre et des particules fluides sur celle-ci, la seconde la continuité de la pression et la troisième l'annulation de la composante normale de la vitesse sur le fond.

En admettant que le vecteur rotation $\omega$ soit vertical et constant $(0,0, \lambda)$ et en se limitant au premier ordre, c'est-à-dire aux petits mouvements [2], on voit facilement que les équations (1) se linéarisent sous la forme:

$$
\begin{gathered}
\frac{\partial i}{\partial t}-\lambda v=\frac{\partial^{2} \Phi}{\partial x \partial t}, \\
\frac{\partial v}{\partial t}+\lambda u=\frac{\partial^{2} \Phi}{\partial y \partial t}, \\
\frac{\partial w}{\partial t}=\frac{\partial^{2} \Phi}{\partial z \partial t}, \\
\frac{\partial \Phi}{\partial t}+\frac{p}{p}+g z+\Gamma=0 \\
\frac{\partial u}{\partial x}+\frac{\partial v}{\partial y}+\frac{\partial w}{\partial z}=0
\end{gathered}
$$

Dans les océans, i représente la composante verticale de la rotation, $2 \omega \sin \varphi$, où $\varphi$ désigne la latitude. Les conditions aux frontières (2) se linéarisent également au premier ordre sous la forme :

$$
\begin{aligned}
& \frac{\partial \zeta}{\partial t}-\left.w\right|_{z=0}=0, \\
& \left|\frac{\partial \Phi}{\partial t}+g \zeta+\Pi\right|_{z=0}=0, \quad \Pi=\frac{p_{a}}{?}+\Gamma, \\
& \left|u \frac{\partial h}{\partial x}+v \frac{\partial h}{\partial y}+w\right|_{z=-h}=0 .
\end{aligned}
$$

Les trois premières relations (3) permettent d'exprimer les composantes de la vitesse en fonction de $\Phi$, soit :

$$
\begin{aligned}
\left(\frac{\partial^{2}}{\partial l^{2}}+\lambda^{2}\right) u & =\frac{\partial}{\partial t}\left(\frac{\partial^{2} \Phi}{\partial x \partial t}+\lambda \frac{\partial \Phi}{\partial y}\right) \\
\left(\frac{\partial^{2}}{\partial t^{2}}+\lambda^{2}\right) v & =\frac{\partial}{\partial t}\left(\frac{\partial^{2} \Phi}{\partial y \partial t}-\lambda \frac{\partial \Phi}{\partial x}\right) \\
w & =\frac{\partial \Phi}{\partial z}
\end{aligned}
$$

$\Phi$ est en effet définie à une fonction d'espace près.

En éliminant ensuite les composantes $u, v, w$, du système formé par ces relations (5) et l'équation de continuité, on obtient l'équation d'onde suivante pour $\Phi$ :

$$
\frac{\partial^{2}}{\partial t^{2}}(\Delta \Phi)+\lambda^{2} \frac{\partial^{2} \Phi}{\partial z^{2}}=0
$$

Quant aux conditions (4), elles donnent, en éliminant $\zeta$ et $w$ et en supposant la profondeur constante,

$$
\left.\begin{array}{rl}
\frac{\partial^{2} \Phi}{\partial t^{2}}+g \frac{\partial \Phi}{\partial z}+\left.\frac{\partial I I}{\partial t}\right|_{z=0} & =0, \\
\left.\frac{\partial \Phi}{\partial z}\right|_{z=-\hbar} & =0 .
\end{array}\right\}
$$

La fonction $\Phi$ doit donc satisfaire au système (6), (7); la dénivellation, la pression et la vitesse s'en déduisent ensuite par:

$$
\begin{aligned}
& \zeta=-\frac{1}{g}\left|\frac{\partial \Phi}{\partial t}+\Pi\right|_{z=0}, \\
& p=p_{t}-\rho\left(g z+\frac{\partial \Phi}{\partial t}+\Pi\right),
\end{aligned}
$$


et par (5). Le mouvement est rotationnel; en effet, on obtient immédiatement de (5) et (6) :

$$
\left(\frac{\partial^{2}}{\partial t^{2}}+\lambda^{2}\right)\left(\frac{\partial}{\partial t} \overrightarrow{\operatorname{rot} u-\lambda} \frac{\partial \vec{u}}{\partial z}\right)=0
$$

lorsque $\lambda \rightarrow 0$, on retrouve les relations classiques.

\section{ONDES LIBRES PÉRIODIQUES}

Des ondes périodiques et à crêtes horizontales ou cylindriques (à deux dimensions) sont représentées par une fonction $\Phi$ de la forme :

$$
\Phi=\varphi(x, z)-i \omega t, \quad \sigma=\frac{2 \pi}{\mathrm{T}}
$$

ò̀ o désigne la fréquence et $\mathbf{T}$ la période.

Supposons que la pression atmosphérique et le potentiel perturbateur n'aient pas d'action : $\Pi=0$. On a dans ce cas des ondes libres et la fonction $९$ obéit au système :

$$
\begin{aligned}
& \sigma^{2} \frac{\partial^{2} \varphi}{\partial x^{2}}+\left(\sigma^{2}-\lambda^{2}\right) \frac{\partial^{2} \varphi}{\partial z^{2}}=0 \\
& \left|g \frac{\partial c}{\partial z}-\sigma^{2} \varphi\right|_{z=0}=0,\left.\quad \frac{\partial \varphi}{\partial z}\right|_{z=-n}=0 .
\end{aligned}
$$

Les solutions qui séparent les variables sont données par :

$$
0=\operatorname{ch} \gamma(h+z) e^{i k x},
$$

et le coefficient $\gamma$ et le nombre d'onde $k$ sont déterminés par les équations

$$
\begin{aligned}
g \gamma \text { th } \gamma h-\sigma^{2} & =0 \\
\gamma^{2}\left(\sigma^{2}-\lambda^{2}\right)-\sigma^{2} k^{2} & =0
\end{aligned}
$$

La première possède une racine réelle $\gamma_{0}$ ct une suite de racines imaginaires pures $\gamma_{n}=i \gamma_{n}^{\prime}$ $(n=1,2, \ldots)$. On a ainsi deux types de solutions :

- Les ondes de surface $(n=0)$, dans lesquelles l'amplitude du mouvement est maximale en surface et décroît exponentiellement avec la profondeur;

-.- Les ondes internes ou stratifièes $(n=1,2, \ldots)$, dans lesquelles le mouvement est divisé en plusieurs couches superposées, séparées par $n$ plans nodaux horizontaux, et comporte $n$ maximums d'amplitude.

On voit sur la seconde équation (13) qu'il faut ensuite distinguer deux cas : $\lambda \leqq \sigma$ ou $\mathrm{T} \leqq \mathrm{T}_{p}$; si $\mathrm{T}_{p}$ désigne la période pendulaire, $\mathrm{T}_{p}=(2 \pi / \lambda)$ (cette période pendulaire vaut environ $17 \mathrm{~h}$ à la latitude de $45^{\circ}$ ).

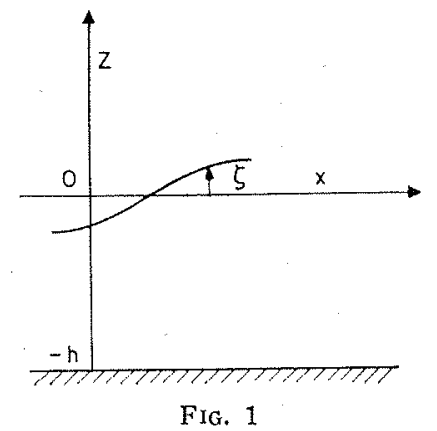

Quand $\mathrm{T}<\mathrm{T}_{p}, k$ est une quantité réelle ou imaginaire en même temps que $\gamma$. Les ondes de surface sont des ondes progressives ou stationnaires par réflexion ( $k$ réel) et les ondes internes des ondes stationnaires exponentielles en $x$ ( $k$ imaginaire).

Quand ' $\mathrm{T}>\mathrm{T}_{p}$, c'est l'inverse : $k$ est une quantité réelle si $\gamma$ est imaginaire, une quantité imaginaire si $\gamma$ est réel. Les ondes de surface sont alors des ondes stationnaires exponentielles en $x$, et les ondes internes des ondes progressives ou stationnaires par réflexion.

Dans le cas des ondes progressives, la fréquence $\sigma$ est une fonction du nombre d'onde $k$ qui est représentée de façon qualitative sur la figure 2 .

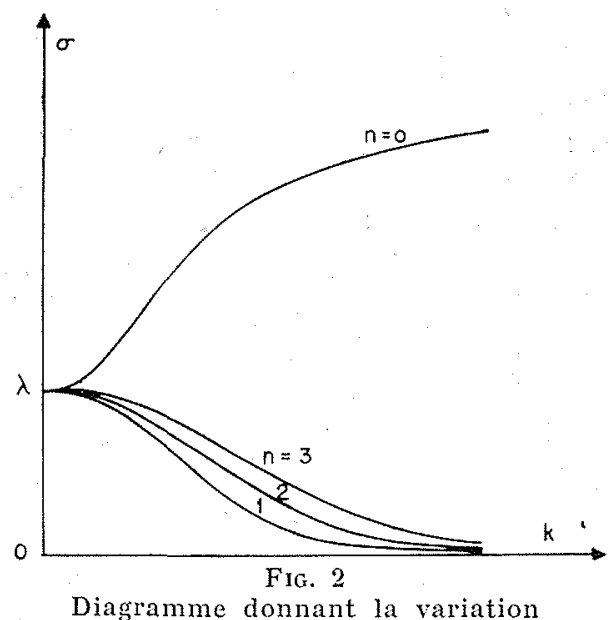

de la fréquence avec le nombre d'onde. 
La vitesse de phase et la vitesse de groupe ont pour expression :

$$
\begin{aligned}
& \mathrm{C}=\frac{\sigma}{k}=\left\{\frac{g \text { th } \gamma h}{\gamma\left[1-\left(\mathrm{T}^{2} / \mathrm{T}_{p}^{2}\right)\right]}\right\}^{1 / 2}, \\
& \mathrm{U}=\frac{d \sigma}{d k} \\
& =\frac{\mathrm{C}}{2}\left(1+\frac{2 \gamma h}{\operatorname{sh} 2 \gamma h}\right) \\
& \left.\times\left\{1+\frac{1}{2} \frac{\mathrm{T}^{2}}{\mathrm{~T}^{2}} \frac{\left[1+\left(2 \gamma h / \mathrm{sh}_{p} \gamma \gamma h\right)\right]}{\left[1-\left(\mathrm{T}^{2} / \mathrm{T}^{2}{ }_{p j}\right)\right]}\right\}^{-1},\right\}
\end{aligned}
$$

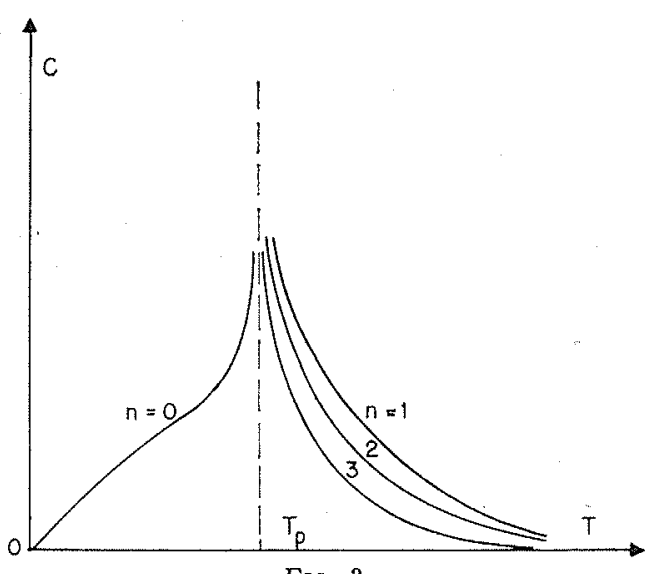

FIG. 3

Diagramme donnant la variation de la vitesse de phase avec la période. où $\gamma$ est lié à la période par la première relation (13). Les figures 3 et 4 montrent comment ces vitesses varient avec la période $T$.

La période pendulaire sépare donc le domaine des périodes en deux régions; le passage d'une région à l'autre se traduit ou bien par l'échange des qualités d'onde de surface et d'onde interne, ou bien par celui des qualités d'onde progressive (ou stationnaire par réflexion) et d'onde stationnaire exponentielle.

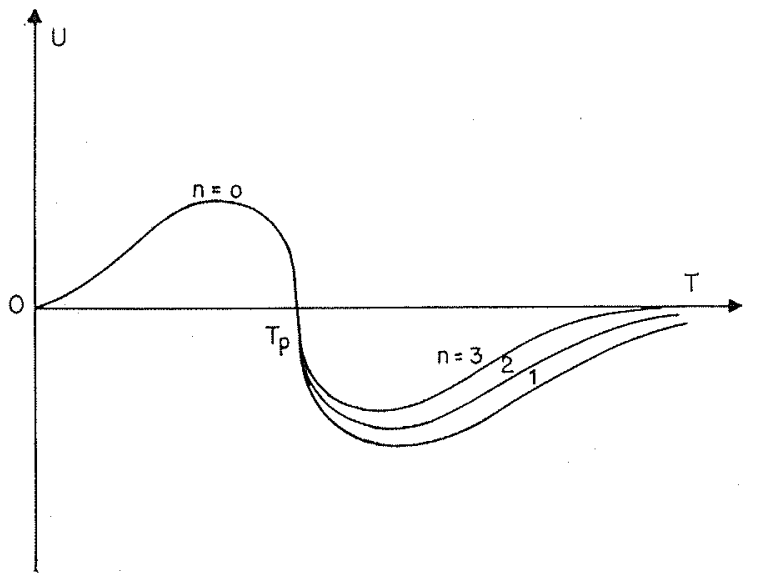

Fig. 4

Diagramme donnant la rariation de la vitesse de groupe avec la période.

\section{ONDES PERIODIQUES PRODUITES PAR UN BATTEUR}

Cherchons maintenant quelles sont les ondes produites par l'agitation périodique d'une paroi verticale dans un bassin tournant de profondeur constante. Si on se limite au problème à deux dimensions et si $x=0$ représente la paroi verticale, le problème à résoudre est le suivant :

Trouver une fonction qui vérifie le système (11) et la condition :

$$
\left.\frac{\partial \varphi}{\partial x}\right|_{z=0}=f(z)
$$

qui exprime que, sur la paroi $x=0$, la vitesse normale à une valeur donnée, égale à $f(z)$. II faut, de plus, ajouter une condition de rayonnement qui s'écrit pour $x>0$ :

$\varphi_{x \rightarrow+\infty} \rightarrow$ une somme d'ondes progressives positives.

Développons comme en l'absence de rotation
[3] la solution o dans la suite des solutions $\varphi_{j}$ précédemment obtenues :

$$
\varphi=\sum_{j=0}^{\infty} c_{j}{ }_{j}
$$

Les $\varphi_{j}$ ont pour expressions :

$$
\begin{aligned}
& \text { si } \mathrm{T}<\mathrm{T}_{p}, \\
& \varphi_{0}=\operatorname{ch} \gamma_{0}(h+z) e^{i \gamma_{0}\left[1-\left(\lambda^{2} / \sigma^{2}\right)\right]^{1 / 2} x,} \\
& \varphi_{n}=\cos \gamma_{n}(h+z) e^{-\gamma_{n}\left[1-\left(\lambda^{2} / \sigma^{2}\right)\right]^{1 / 2} x,} \\
& r=1,2, \ldots \\
& \text { si } \mathrm{T}>\mathrm{T}_{p}, \\
& \varphi_{0}=\operatorname{ch} \gamma_{0}(h+z) e^{-\gamma_{0}\left[\left(\lambda^{2} / 0^{2}\right)-1\right]^{1 / 2}} x, \\
& \varphi_{n}=\cos \gamma_{n}(h+z) e^{\left.i \gamma_{n} \mathrm{~L}\left(\lambda^{2} / \sigma^{2}\right)-1\right] 1 / 2} x, \\
& n=1,2, \ldots
\end{aligned}
$$


où $\gamma_{0}$ et $\gamma_{n}$ désignent les racines de la première équation (13):

$$
\sigma^{2}-g \gamma_{0} \text { th } \gamma_{0} h=0, \quad \sigma^{2}+g \gamma_{n} \operatorname{tg} \gamma_{n} h=0 .
$$

La solution (16) satisfait bien au système (11) et à la condition de rayonnement. La condition (15) s'écrit :

$$
\left.\sum_{j=0}^{\infty} c_{j} \frac{\partial \varphi_{j}}{\partial x}\right|_{x=0}=f(z)
$$

elle est vérifiée s'il est possible de développer $f(z)$ dans la suite de fonctions $\operatorname{ch} \gamma_{0}(h+z)$, $\cos \gamma_{n}(h+z)$, c'est-à-dire si cette suite est orthogonale et fermée. Il est facile de vérifier qu'elle est orthogonale, mais plus difficile de montrer qu'elle est fermée [2], [4]. En admettant ce dernier point, on peut développer $f(z)$ dans cette suite :

$f(z)=f_{0} \operatorname{ch} \%_{0}(h+z)+\sum_{n=1}^{\infty} f_{n} \cos \%_{n}(h+z)$

et les coefficients $f_{f}$ de ce développement sont donnés par: :

$$
\left.\begin{array}{l}
f_{0}=\frac{\int_{-h}^{0} f(z) \operatorname{ch} \gamma_{0}(h+z) d z}{\int_{-h}^{0} \operatorname{ch}^{2} \gamma_{0}(h+z) d z}, \\
f_{n}=\frac{\int_{-h}^{0} f(z) \cos \gamma_{n}(h+z) d z}{\int_{-h}^{0} \cos ^{2} \gamma_{n}(h+z) d z} .
\end{array}\right\}
$$

La condition (18) permet ensuite de déterminer les coefficients $c_{j}$ :

si $\mathrm{T}<\mathrm{T}_{p}$,

$$
\begin{aligned}
& i \vartheta_{0}\left(1-\frac{\lambda^{2}}{\sigma^{2}}\right)^{1 / 2} c_{0}=f_{0}, \\
& -\gamma_{n}\left(1-\frac{\lambda^{2}}{\sigma^{2}}\right)^{1 / 2} c_{n}=f_{n},
\end{aligned}
$$

si $\mathrm{T}>\mathrm{T}_{p}$,

$$
\begin{aligned}
& -\gamma_{0}\left(\frac{\lambda^{2}}{\sigma^{2}}-1\right)^{1 / 2} c_{0}=f_{0} \\
& i \gamma_{n}\left(\frac{\lambda^{2}}{\sigma^{2}}-1\right)^{1 / 2} c_{n}=f_{n}
\end{aligned}
$$

Ainsi, pour des périodes inférieures à la période pendulaire, le phénomène est qualitativement le même qu'en l'absence de rotation; les ondes produites sont une suite d'ondes internes stationnaires dont l'amplitude décroît exponentiellement à partir de la paroi génératrice, et une onde de surface progressive qui se propage à l'infini avec une amplitude constante. Inversement, pour des périodes supérieures à la période pendulaire, les ondes produites sont une suite d'ondes internes progressives qui se propagent à l'infini avec une amplitude constante, et une onde de surface stationnaire dont l'amplitude décroît exponentiellement à partir de la paroi génératrice.

\section{ONDES PERIODIQUES \\ DANS UN CANAL DE PROFONDEUR CONSTANTE}

Les résultats précédents caractérisent des ondes à crêtes horizontales. Les solutions représentant des ondes libres dans un canal sont nécessairement des ondes à crêtes exponentielles et ondulées, c'est-à-dire des ondes de Kelvin ou de Poincaré [5]. Le problème étant tridimensionnel, la fonction $\Phi$ est maintenant de la forme

$$
\Phi=p(x, y, z) e^{-i \omega t}, \quad\left(\sigma=\frac{2 \pi}{\mathrm{T}}\right)
$$

et la fonction o obéit au système :

$$
\begin{gathered}
\sigma^{2} \Delta \varphi-\lambda^{2} \frac{\partial^{2} \varphi}{\partial z^{2}}=0 \\
g \frac{\partial \varphi}{\partial z}-\left.\sigma^{2} \varphi\right|_{z=0}=0,\left.\quad \frac{\partial \varphi}{\partial z}\right|_{z=-h}=0
\end{gathered} \mid
$$

Supposons le canal limité par les parois $y=0, a$; il faut ajouter les conditions

$$
\left.v\right|_{y=0, a}=0
$$

qui, en vertu de (5), sont des conditions de dérivée oblique pour $\varphi$ :

$$
\left|i \sigma \frac{\partial \varphi}{\partial y}+\lambda \frac{\partial \varphi}{\partial x}\right|_{y=0, a}=0 \text {. }
$$

II est facile de former une double suite de solutions $\varphi_{j l}(j, l,=0,1, \ldots)$ du système (23), (24) qui est la suivante pour $x>0$ (condition de rayonnement) :

$$
\begin{aligned}
\varkappa_{00} & =\operatorname{ch} \gamma_{0}(h+z) e^{i \gamma_{0} m-\gamma_{0}(\lambda / \sigma) y}, \\
\oiiint_{0 m} & =\operatorname{ch} \gamma_{0}(h+z) \\
& \times\left(\frac{m \pi}{a} \cos \frac{m \pi y}{a}+\frac{i \lambda k_{o m}}{\sigma} \sin \frac{m \pi y}{a}\right) e^{-k_{o m}{ }^{2}}
\end{aligned}
$$


avec

$$
k_{0 m}^{2}=\frac{m^{2} \pi^{2}}{a^{2}}-\gamma_{0}^{2}\left(1-\frac{\lambda^{2}}{\sigma^{2}}\right)
$$

$k_{0 m}$ imaginaire pour

$$
m<\gamma_{0} \frac{a}{\pi}\left(1-\frac{\gamma^{2}}{\sigma^{2}}\right)^{1 / 2}
$$

et

$$
\begin{aligned}
\varphi_{n 0}= & \cos \gamma_{n}(h+z) e^{-\gamma_{\mathrm{n}} x-i \gamma_{n}(\lambda / \sigma) y} \\
\varphi_{n m}= & \cos \varphi_{n}(h+z) \\
& \times\left(\frac{m \pi}{a} \cos \frac{m \pi !}{a}+\frac{i \lambda k_{m m}}{\partial} \sin \frac{m \pi ! j}{a}\right) e^{-k_{n+m} x}
\end{aligned}
$$

avec

$$
k_{n m}^{2}=\frac{m^{2} \pi^{2}}{a^{2}}+\gamma^{2}{ }_{n}\left(1-\frac{\lambda^{2}}{\sigma^{2}}\right),
$$

$k_{n m}$ imaginaire pour

$$
m<\gamma_{n} \frac{a}{\pi}\left(\frac{\lambda^{2}}{\sigma^{2}}-1\right)^{1 / 2}
$$

et

$$
\begin{gathered}
\sigma^{2}+g \%_{n} \operatorname{tg} \gamma_{n} h=0 \\
(n=1,2, \ldots), \quad(m=1,2, \ldots) .
\end{gathered}
$$

Ces solutions sont soit des ondes de Kelvin à crêtes exponentielles en $y$, soit des ondes de Poincaré à crêtes ondulées en $y$. Quand la période est inférieure à la période pendulaire, les ondes progressives sont des ondes de surface. L'une poo, est une onde de Kelvin; les autres $\varphi_{0 m}$ avec $\quad m<\gamma_{0} \frac{a}{\pi}\left(1-\frac{\lambda^{2}}{\sigma^{2}}\right)^{1 / 2}$

sont des ondes de Poincaré en nombre limité, quand elles existent. Quand la période est supérieure à la période pendulaire, les ondes progressives sont l'onde de Kelvin $\vartheta_{00}$ qui est une onde de surface, puis les ondes de Poincaré '? avec $\quad m<\gamma_{n} \frac{a}{\pi}\left(\frac{\lambda^{2}}{\sigma^{2}}-1\right)^{1 / 2}$

qui sont des ondes internes. Là aussi, la rolation intervient de facon essentielle sur la forme et la propagation dés ondes liquides de gravité, surtout pour les périodes supérieures à la période pendulaire. Ces résultats ne sont évidemment plus valables si les amplitudes sont grandes ou les périodes petites; les approximations d'ordre supérieur et la viscosité interviennent alors nécessairement.
[1] M. Brillovin et J. Covxomis : Oscillations d'un liquide pesant dans un bassin cylindrique en rotation. - Gauthier-Villars, Paris (1933).

[2] J.J. Stoken : Water waves. -- Interscience Publishers, New York (1957).

[3] T, H. Havelock: Forced surface waves on water. Phil. Mag. (1929), 8, 51.
4] M. Weinstern : Sur un problème aux limites dans une bande indéfinie. - Comptes rendus Acad. Sci. $(1927), 184$, p. 497.

[5] H. Poncaré : Leçons de mécarique céleste, t. III. Gauthier-Villars, Paris (1910).

IMPORTANT BUREAU D'ETUDES TRAVAUX PUBLICS

recherche pour poste outre-mer :

INGÉNIEURS EXPÉRIMENTÉS HYDROGEOLOGUES, HYDROLOGUES et HYDRAULICIENS

Ecrire à B 306 PUBLIPRESS, 36, bd Bonne-Nouvelle, Paris (2 ${ }^{\mathrm{e}}$ ) 